\title{
EPSL
}

ELSEVIER

\section{Calibration of magnetic granulometric trends in oceanic basalts}

\author{
J. Gee ${ }^{\mathrm{a}, *}$, D.V. Kent ${ }^{\mathrm{b}, \mathrm{c}}$ \\ ${ }^{a}$ Scripps Institution of Oceanography, La Jolla, CA 92093-0215, USA \\ ${ }^{b}$ Lamont Doherty Earth Observatory, Palisades, NY 10964, USA \\ ${ }^{c}$ Department of Geological Sciences, Rutgers University, Piscataway, NJ 08854-8066, USA
}

Received 9 October 1998; revised version received 22 February 1999; accepted 21 May 1999

\begin{abstract}
The validity of magnetic granulometric estimates relies heavily on the ability to distinguish ultrafine particles from coarser grains. For example, populations with dominantly superparamagnetic (SP) or multidomain (MD) grains both are characterized by low remanence and coercivity, and distinguishing these endmembers may provide valuable clues to the origin of magnetization in the intervening stable single domain (SD) size range. The natural grain size variations associated with variable cooling rates in submarine lavas provide a rare opportunity for examining progressive changes in average magnetic grain size, from SP-SD mixtures in submarine basaltic glass to SD-MD mixtures in flow interiors. Based on microanalysis and rock magnetic measurements on pillow basalt samples dredged from the flanks of the Mid-Atlantic Ridge (ages $<1 \mathrm{Ma}$ to $70 \mathrm{Ma}$ ), a model of preferential dissolution with time of the finest-grained titanomagnetites has recently been suggested as the major process contributing to long-term temporal changes in remanent intensity of mid-ocean ridge basalts. We evaluated the local and long-term temporal trends in effective magnetic grain size predicted by this model using hysteresis data from a large number of submarine basalt samples which span a range of ages from $\sim 0$ to $\sim 122 \mathrm{Ma}$. Specimens were systematically taken along transects perpendicular to the chilled margin of each sample. The large number of data ( $\sim 750$ loops) and the inferred progressive change in grain size approaching the chilled margin allow recognition of mixing trends between MD and SD grains and between SD and SP grains on a Day-plot. These trends in hysteresis parameters are crucial to resolving the inherent, but frequently overlooked, ambiguity in inferring grain size from hysteresis parameters. We illustrate that two additional rock magnetic tests (warming of a low-temperature isothermal remanence and hysteresis loop shapes) often used to address these ambiguities are inconclusive, requiring some independent knowledge of whether SP or MD grains are likely to be present. Even with a considerably larger data set the substantial intrasample variability in oceanic basalts precludes recognition of any systematic trend in magnetic grain size with age. () 1999 Elsevier Science B.V. All rights reserved.
\end{abstract}

Keywords: magnetization; mid-ocean ridge basalts; grain size; titanomagnetite; hysteresis; coercivity

\section{Introduction}

Fine-grained submarine basalts are commonly regarded as the dominant source for lineated marine

* Corresponding author. Tel.: +1-619-534-4707; Fax: +1-619534-0784; E-mail: jsgee@ucsd.edu magnetic anomalies [1]. Rapid cooling results in magnetic grain sizes ranging from submicron single domain to superparamagnetic (SD-SP) grains in and near the glassy margin [2-4] to skeletal grains tens of microns in size that are presumably pseudosingle domain-multidomain (PSD-MD) in pillow/flow interiors. The strong grain size dependence of mag- 
netic properties results in substantial changes in magnetic parameters over spatial scales that can be on the order of a centimeter or less $[5,6]$. The typically fine-grained nature of titanomagnetites in submarine lavas thus presents two problems for understanding processes, such as low-temperature alteration, that are likely to be important in the temporal evolution of crustal magnetization. First, the range of magnetic grain sizes in submarine lavas may encompass both small (SD-SP) and larger (PSD-MD) grains on a centimeter scale in single flows, raising the possibility of aliasing especially with small number of samples and inadequate documentation. In any given specimen, bulk magnetic properties may be dominated by a small number of larger grains and may not be representative of the smaller grains likely to be the dominant carriers of magnetic remanence $[7,8]$. Second, the fine grain size has made direct analysis (e.g., by microprobe or through magnetic separation) of titanomagnetite composition or degree of alteration difficult, particularly for the finest grains which are likely to be carrying the bulk of the remanence.

Based on microanalysis and rock magnetic measurements, $\mathrm{Xu}$ et al. [9] have recently suggested a model of grain size dependent alteration in submarine basalts which they argue is the major process contributing to long-term temporal changes in remanent intensity of mid-ocean ridge basalts (MORB) [10]. Specifically, based on four pillow basalt samples dredged from the flanks of the Mid-Atlantic Ridge (ages from $<1 \mathrm{Ma}$ to $70 \mathrm{Ma}$ ), Xu et al. suggest that the finest-grained SP-SD grains are subject to preferential dissolution (transformation to nonmagnetic phases) with time. The principal evidence they cite for a systematic increase in effective magnetic grain size (reflecting dissolution of the finest grains) with time in these samples is trends in hysteresis parameters. Supporting evidence cited for a decrease in amount of fine-grained material with age comes from low-temperature experiments and the degree of constriction in hysteresis loops. In contrast to submicron grains which simply dissolve, $\mathrm{Xu}$ et al. [11] have shown that larger grains undergo single-phase maghemitization (cation loss) with a possible later stage of vacancy ordering.

The model of dissolution of fine-grained TM outlined above provides an attractive mechanism for temporal variations in the remanence of oceanic basalts. In particular, Xu et al. [9] suggest that the preferential loss of fine-grained TM, the most potent carrier of remanence in MORB, is responsible for NRM decay with a concomitant increase in effective magnetic grain size with age. We evaluate the temporal trends in effective magnetic grain size predicted by Xu et al. [9] by using hysteresis data from a large number of submarine basalt samples which span a range of ages from $\sim 0$ to $\sim 122 \mathrm{Ma}$ and from which specimens were systematically taken along transects perpendicular to the chilled margin of each sample. The systematic sampling and large number of data ( $\sim 750$ specimens) allow recognition of mixing trends from MD-SD and SD-SP that are crucial to removing the inherent, but frequently overlooked, ambiguity in inferring grain size from hysteresis parameters. We illustrate that two additional rock magnetic tests (warming of a low-temperature IRM and hysteresis loop shapes) often used to address these ambiguities are inconclusive, requiring some independent knowledge of whether SP or MD grains are likely to be present. Re-evaluation of the data of $\mathrm{Xu}$ et al. [9] suggests an alternative interpretation, whereby the oldest of their four samples in fact contains the finest magnetic grains. Nevertheless, we find that even with a considerably larger data set the substantial intrasample variability in oceanic basalts precludes recognition of any systematic trend in magnetic grain size with age.

\section{MD-SD and SD-SP mixing trends on a Day-plot}

We generated magnetic hysteresis data for specimens along systematic sampling transects relative to the chilled margin for three sets of MORB samples. These sampling transects include: (1) a subset of the essentially zero age dredge samples from the southern East Pacific Rise (SEPR) studied by Gee and Kent [12]; (2) 29 samples from 14 dredges with ages $0-1 \mathrm{Ma}$ from near $10^{\circ} \mathrm{N}$ on the EPR (Phoenix expedition; [13]); and (3) 24 samples from 10 DSDP drill-core sites with ages of 0.2-122 Ma. For each of these samples, a slab was cut approximately perpendicular to the glassy margin of the flow/pillow. Each slab was then subsampled to provide a tran- 
sect of specimens (at $\sim 1 \mathrm{~cm}$ resolution) orthogonal to the cooling margin. The outermost (a) specimen was often subdivided for SEPR and Phoenix samples in an effort to better document the large gradient in magnetic properties near the chilled margin. Hysteresis parameters were determined on $\sim 30 \mathrm{mg}$ chips with an alternating gradient force magnetometer (Micromag 2900) using a maximum field of 1.0 $\mathrm{T}$. The ratios of saturation remanence to saturation magnetization $\left(M_{\mathrm{rs}} / M_{\mathrm{s}}\right)$ were calculated following a standard correction for the high field paramagnetic slope from 0.7 to $1.0 \mathrm{~T}$. The magnetic hysteresis data for all the specimens are summarized on a bilogarithmic Day-diagram [14] of $M_{\mathrm{rs}} / M_{\mathrm{s}}$ versus the ratio of remanent coercivity to coercivity $\left(B_{\mathrm{rc}} / B_{\mathrm{c}}\right)$ (Fig. 1).

The slab data show a substantial range of hysteresis properties overall (Fig. 1A). Most of the data lie along a broad trend compatible with mixtures of typical MD and SD grains. Because $M_{\mathrm{rs}} / M_{\mathrm{s}}$ ratios are commonly $>0.5$ (the theoretical limit for uniaxial anisotropy; [15]), we adopt a SD endmember with theoretical hysteresis values $\left(M_{\mathrm{r}} / M_{\mathrm{s}}=0.83-\right.$ $\left.0.87 ; B_{\mathrm{rc}} / B_{\mathrm{c}}=1.04-1.08\right)$ compatible with cubic anisotropy [16]. Theoretical considerations provide only general limits on the hysteresis parameters for true MD grains $\left(M_{\mathrm{rs}} / M_{\mathrm{s}}<0.05 ; B_{\mathrm{rc}} / B_{\mathrm{c}}>4 ;[17]\right)$;

Fig. 1. Bilogarithmic Day-plots of magnetic hysteresis data from submarine basalts. (A) Results from lavas systematically sampled with respect to the chilled margin. Slabs were cut approximately perpendicular to the chilled margin and subsampled at approximately a $1 \mathrm{~cm}$ interval. (B) Representative slab samples illustrating the progression of hysteresis parameters as the chilled margin (large symbols) is approached. Lines connect successively deeper samples. (C) Results from isolated submarine basalt samples. Isolated samples include SEPR 'B' specimens systematically taken 1-2 cm from the chilled margin [12]. Samples from $\mathrm{Xu}$ et al. [9] are from a depth of $>2 \mathrm{~cm}$ from the chilled margin. No information on the distance from the chilled margin is available for samples from DSDP/ODP sites $[20,21]$. Locations of theoretical single domain (SD) endmember are based on dominant cubic anisotropy [16]. Range of possible mixing trends from multidomain (MD) material based on variability in hysteresis parameters $\left(1.7<B_{\mathrm{rc}} / B_{\mathrm{c}}<3.1\right.$ at $M_{\mathrm{rs}} / M_{\mathrm{S}}$ of approximately 0.1$)$ for coarse-grained synthetic and crushed natural (titano)magnetites [18]. Superparamagnetic (SP) endmember based on hysteresis parameters from a glassy basalt sample (this study). Mixing trends are shown schematically by heavy grey lines. Nonlinear mixing trend between MD and SD after [18]. however, coarse natural and synthetic grains exhibit a range of hysteresis parameters extending nearly an order of magnitude beyond these limits. Clearly, no single MD endmember can be identified, and published hysteresis data from coarse-grained (titano)magnetites form a broad swath on the Day-diagram. For comparison with the range of hysteresis data in this study, we use observed $B_{\mathrm{rc}} / B_{\mathrm{c}}$ coordi-
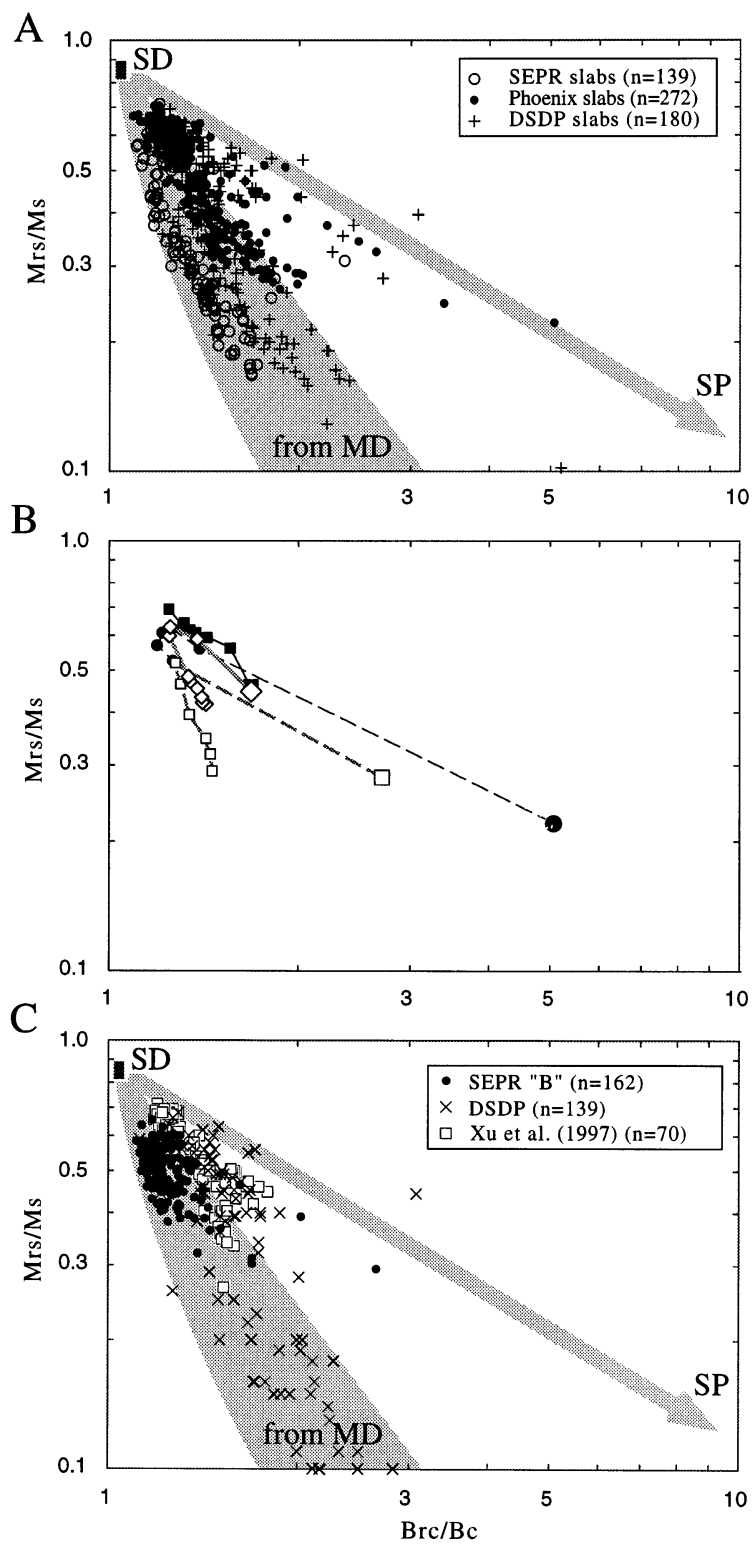
nates of $\sim 1.7$ to 3.1 at an $M_{\mathrm{rs}} / M_{\mathrm{s}}$ value of 0.1 [18] to provide approximate values for the swath of mixing trends involving these coarse MD grains and the SD endmember (Fig. 1).

Systematic variations in hysteresis parameters within slab samples allow us to relate the trends to monotonic changes in magnetic grain size, as inferred from distance to the chilled margin. Specifically, samples from the interior of flows have more MD-like hysteresis parameters, with a serial progression towards the SD endmember as the cooling margin is approached. Note that mixtures between SD and MD grains depart from a linear trend on the bilogarithmic Day-plot as the SD endmember is approached, similar to the trend documented for synthetic (hydrothermally grown) and crushed natural magnetites [18]. The considerable variability in hysteresis parameters for MD material [18] as well as the possible influence of uniaxial anisotropy (possibly arising from compositional variability) and more complex magnetic structures (e.g., vortex states; [17]) may contribute to the relatively large scatter along the MD-SD mixing trend.

A subset of the hysteresis data shows much higher $B_{\mathrm{rc}} / B_{\mathrm{c}}$ values at a given $M_{\mathrm{rs}} / M_{\mathrm{s}}$, apparently forming a second trend on the Day-plot (Fig. 1A). These values are invariably found to be associated with samples near the chilled margin, and therefore presumably with the finest magnetic grain sizes. This association is confirmed by the detailed trajectory of hysteresis parameters, which typically has a systematic progression towards the SD endmember but then hooks towards a presumed endmember with low $M_{\mathrm{rs}} / M_{\mathrm{s}}$ but much higher $B_{\mathrm{rc}} / B_{\mathrm{c}}$ (Fig. 1B). Given the independent evidence for approach to very fine grain sizes (i.e., glass), we hypothesize that this second trend represents mixing between SD and SP grains [4]. Although an assemblage of pure SP grains has, by definition, no remanence $\left(M_{\mathrm{rs}} / M_{\mathrm{S}}=0\right)$ and no coercivity $\left(B_{\mathrm{rc}} / B_{\mathrm{c}}\right.$ undefined), any naturally occurring distribution of predominantly $\mathrm{SP}$ grains will likely have some stable SD grains resulting in a finite remanence and coercivity. For the empirical SP endmember for this trend, we therefore use hysteresis parameters $\left(M_{\mathrm{rs}} / M_{\mathrm{s}}=0.017, B_{\mathrm{rc}} / B_{\mathrm{c}}=97\right)$ for a glassy basalt sample with the highest $B_{\mathrm{rc}} / B_{\mathrm{c}}$ value from our sample collection. As with the SD-MD mixing trend, mixtures of SD and SP material may well be nonlinear on the bilogarithmic Day-plot. However, a simple linear mixing trend adequately represents the changes in hysteresis parameters in most near-margin samples (Fig. 1B). Further indications of the influence of SP in this trend are discussed below.

Hysteresis data for a series of near-margin specimens from axial dredges along the southern East Pacific Rise [12] highlight the substantial gradient in magnetic properties near the chilled margin (Fig. 1B). Although these specimens were taken at a uniform distance $(1-2 \mathrm{~cm})$ from the chilled margin, hysteresis data plot along both the MD-SD trend (with $M_{\mathrm{rs}} / M_{\mathrm{s}}$ as low as 0.30 ) as well as on the SDSP trend $\left(M_{\mathrm{rs}} / M_{\mathrm{s}}=0.3\right.$ but $\left.B_{\mathrm{rc}} / B_{\mathrm{c}}=2.8\right)$. Clearly, significant changes in hysteresis properties may occur over distances as small as a few millimeters near the flow margins. We note that the composition of groundmass titanomagnetites in these samples is remarkably uniform (modal $x^{\prime}=0.67$; where $x^{\prime}$ is the modified ulvöspinel content of Stormer [19]) [12]. Recognition of both the SD-SP and MD-SD trends in this limited sample set therefore suggests that compositional variability is not responsible for these two mixing trends.

Recognition of the MD-SD and SD-SP trends on the Day-diagram allows more complete granulometric characterization of discrete sample data, where independent documentation of grain size variations may not be available. For example, Fig. 1C shows hysteresis data from several North Atlantic DSDP Leg 49 sites [20] and ODP Hole 801C [21] where no indication was given as to distance from a chilled margin. These data are generally compatible with the two mixing trends described above. The majority of these drill-core samples lie along the mixing trend between MD and SD with only a few samples apparently along the SD-SP trend. The paucity of samples with significant SP contribution is not unexpected, as such samples occur only in a narrow band (typically 1-2 cm; Fig. 1B) near the glassy margin. Similarly, we show the data from $\mathrm{Xu}$ et al. [9] where it is also uncertain exactly where they were obtained with respect to a chilled margin. Most of their samples lie along the MD-SD mixing trend. However, it is interesting that the oldest of the four dredge samples studied by $\mathrm{Xu}$ et al. [9] includes samples which apparently lie along the SD-SP trend $\left(M_{\mathrm{rs}} / M_{\mathrm{s}} \sim 0.42\right.$, 
$\left.B_{\mathrm{rc}} / B_{\mathrm{c}} \sim 1.8\right)$ suggesting that this sample may contain the finest magnetic grains rather than the largest grain sizes as inferred by Xu et al. [9].

The shapes of hysteresis loops vary systematically with grain size, and hence with position on the mixing trends on the bilogarithmic Day-plot. Samples with more MD-like hysteresis parameters along the MDSD trend show narrow hysteresis loops (Fig. 2A) although all of the MORB samples investigated here apparently have a significant proportion of SD or PSD grains $\left(M_{\mathrm{rs}} / M_{\mathrm{s}}\right.$ typically $\left.>0.2\right)$. As samples approach the SD endmember, hysteresis loops become more square shouldered (Fig. 2B), with $M_{\mathrm{rs}} / M_{\mathrm{s}}$ values frequently above 0.5 that are indicative of cubic (magnetocrystalline) anisotropy $[15,16]$.

Because the approach to saturation for SP grains varies considerably with particle size [22], the trend between SD and SP is perhaps best determined empirically. Hysteresis loops along this mixing trend are characterized by reduced $M_{\mathrm{rs}} / M_{\mathrm{S}}$ (Fig. 2C), with increasingly narrow loops (lower $B_{\mathrm{c}}$ ) reflecting the addition of greater volumetric amounts of SP material. There is sometimes moderate constriction in the loops which potentially contains additional information about the SP contribution $[9,22]$. Our empirical endmember SP sample is obviously a mixture of some SD grains since it has a finite remanence. Nevertheless, we find the hallmark trait of a large SP contribution in our endmember sample: low $M_{\mathrm{r}} / M_{\mathrm{s}}$ (as with MD) but high $B_{\mathrm{rc}} / B_{\mathrm{c}}$ (arising from the presence of a small amount of SD grains with high coercivity; Fig. 2D). The shape of the hysteresis loop is compatible with SP (Langevin function) but, although constriction is difficult to measure, is not noticeably wasp-waisted.

\section{Discrimination of SP and MD grains}

The data above illustrate the advantages of systematic sampling within a submarine lava to facilitate interpretation of magnetic granulometry data. Although complex flow morphologies (e.g., multiple quench zones) may sometimes result in more complex relationships, the distance from the chilled margin provides a robust indicator of relative changes in magnetic grain size. Indeed, the natural grain size variations associated with variable cooling rates in submarine lavas provide an ideal setting for examining progressive changes in average magnetic grain size, from SP-SD mixtures in submarine basaltic glass to SD-MD mixtures in flow interiors. However, magnetic granulometry studies are commonly conducted on submarine lava samples without careful documentation of the relationship to chilled margins. Moreover, more general applications of magnetic granulometry most often do not have the benefit of an independent means of assessing relative grain size variations. In these cases, the validity of magnetic granulometric estimates relies heavily on the ability to distinguish ultrafine SP particles from coarser (PSD-MD) grains. We examine here two common tests for distinguishing the presence of SP grains: constriction of magnetic hysteresis loops and the temperature dependence of a low-temperature IRM.

\subsection{Wasp-waisted loops and SP contribution}

Mixtures of SD and SP grains may result in pronounced constriction of hysteresis loops, provided the two populations contribute comparable amounts of magnetization [22,23]. As wasp-waisted loops may also result from a mixture of two minerals with different coercivities [24,25] or from oxidation [26], some independent verification of the presence of ultrafine material is necessary to establish that constriction is due to the presence of a significant SP fraction. In a study of submarine basaltic glasses, Pick and Tauxe [3] reported a maximum grain size of $\sim 20 \mathrm{~nm}$ (from TEM observations) and concluded that the wasp-waisted hysteresis loops in these glass samples resulted from the mixture of volumetrically similar amounts of SD and SP grains. More recently, $\mathrm{Xu}$ et al. [9] have suggested that the degree of constriction of hysteresis loops may be used to quantify the amount of SP material in submarine basaltic samples. Xu et al. noted a general decrease in the degree of loop constriction with age that they interpreted in terms of a decreasing contribution of SP material in their older samples.

If our mixing model is correct and the constriction of loops is a measure of SP in submarine lavas, as suggested by $\mathrm{Xu}$ et al. [9], then it follows that one would expect most wasp-waisted loops to occur on SD-SP trend and that no constriction would be observed for samples with progressively more coarse 
B
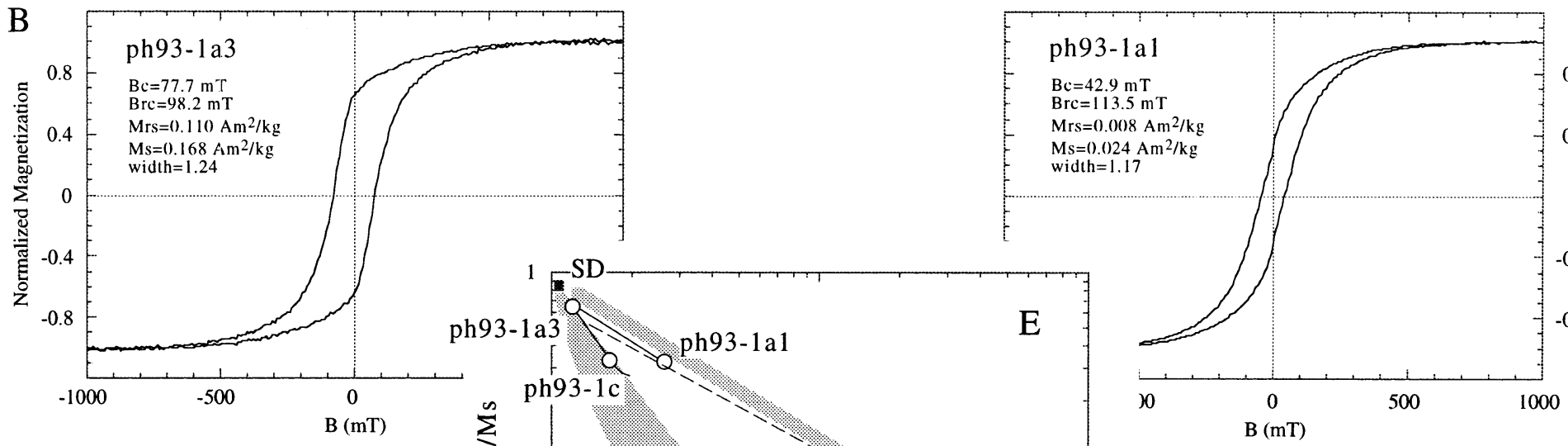

C

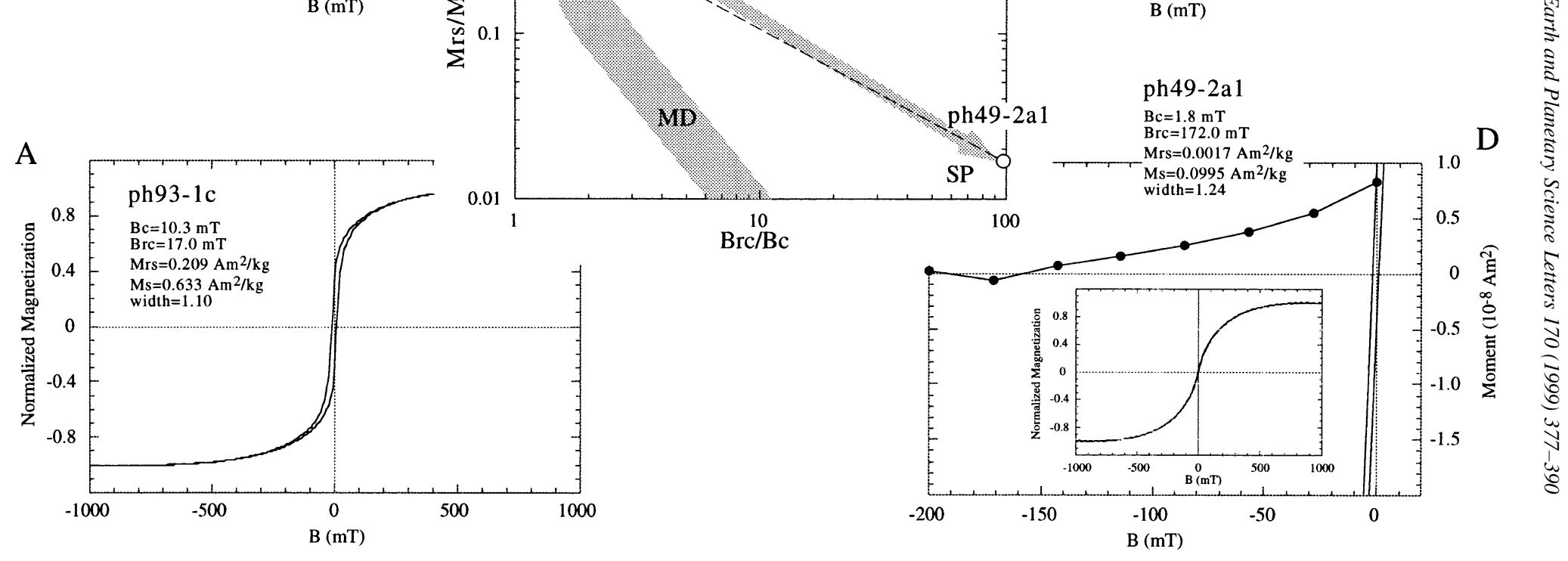

Fig. 2. Representative hysteresis loops illustrating variations in shape along (A,B) the MD-SD and (C,D) SD-SP mixing trends. Hysteresis parameters and wasp-waisted factor are given for each sample (see text for explanation). Note that only the low field region (not slope corrected) is shown in (D), together with the backfield remanence curve (filled circles). The inset for this figure shows the entire (slope corrected) loop. Schematic mixing trends (grey lines) and hysteresis parameters for individual samples are shown in (E). Light solid (dashed) lines connect successive samples with respect to the chilled margins of samples ph93-1 (ph49-2). Note the expan ded range relative to Fig. 1. Broad multidomain (MD) region at $M_{\mathrm{rs}} / M_{\mathrm{s}}<0.1$ based on range of hysteresis parameters of coarse-grained synthetic (hydrothermal) and crushed natural (titano)magnetites [18]. Other symbols as in Fig. 1. 


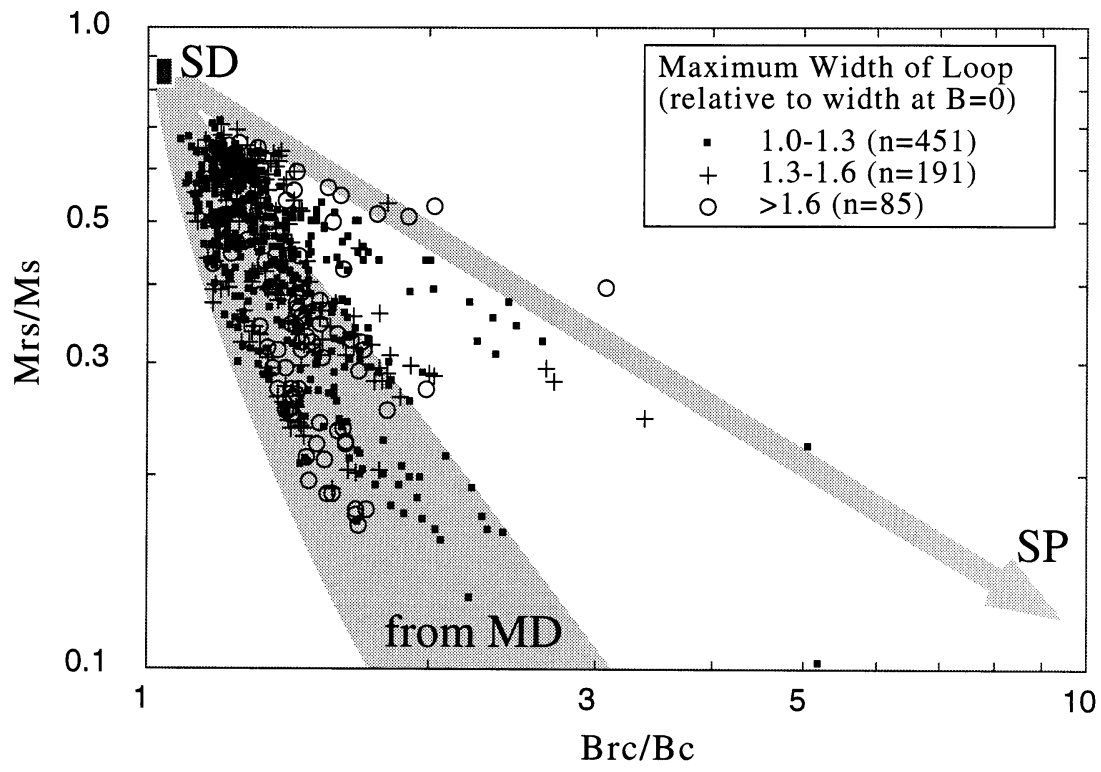

Fig. 3. Day-plot showing degree of constriction of hysteresis loops. The degree of constriction is given by the ratio of the maximum width of the hysteresis loop to the width at zero magnetization [9]. Mixing trends and additional symbols as in Fig. 1.

MD-like grains. In order to test this prediction, we have calculated the degree of constriction (the waspwaisted factor in [9]) for the hysteresis loops in the present study (Fig. 3). Over the range of field values not used in the slope correction $( \pm 0.7 \mathrm{~T})$, we calculated the ratio of the maximum width of the loop relative to the width at zero magnetization (i.e., $2 H_{\mathrm{c}}$ ). From analysis of $\sim 750$ hysteresis loops, it appears that there is no simple relationship between the degree of loop constriction (as defined in [9]) and the position on the mixing trends on the Day-diagram (Fig. 3) or with relative grain size inferred from the position of a sample with respect to the chilled margin. Samples with low wasp-waisted factors, including the sample taken here as the empirical SP endmember, occur along the SD-SP mixing trend. Moreover, samples with high wasp-waisted factors are not limited to the SD-SP trend, but include specimens from the interiors of pillows that plot on the MD-SD mixing trend well away from the SD endmember.

\subsection{Low-temperature measurements}

Remanence measurements during the warming of an IRM acquired at low temperature are commonly used as a means of assessing the contribution of SP grains. For example, the unblocking of an IRM acquired at liquid nitrogen temperature should allow estimation of the proportion of SP grains with blocking temperatures between $77 \mathrm{~K}$ to room temperature. This test has proven useful in quantifying the SP contribution in submarine basaltic glass, where independent TEM observations indicate a grain size distribution centered near the SD-SP boundary [2,3]. Using this same technique, $\mathrm{Xu}$ et al. [9] noted pronounced IRM decay in young submarine basalt samples but little remanence loss in older samples, leading them to suggest that the finest grain size fraction had been preferentially removed in the older samples. As with the degree of constriction of hysteresis loops, we illustrate below that the results from such low-temperature data can also be ambiguous.

Although unblocking of SP grains provides one cause for decay of a low-temperature IRM during warming, significant decay is also observed in samples unlikely to have any detectable SP material. Fig. 4A shows the decay of a low-temperature IRM for such a basalt sample from the northern EPR. Both microprobe analyses $\left(0.49<x^{\prime}<0.60\right)$ and Curie temperatures $\left(\sim 150^{\circ} \mathrm{C}\right.$; see [7] for additional rock magnetic data on this sample) suggest that this 

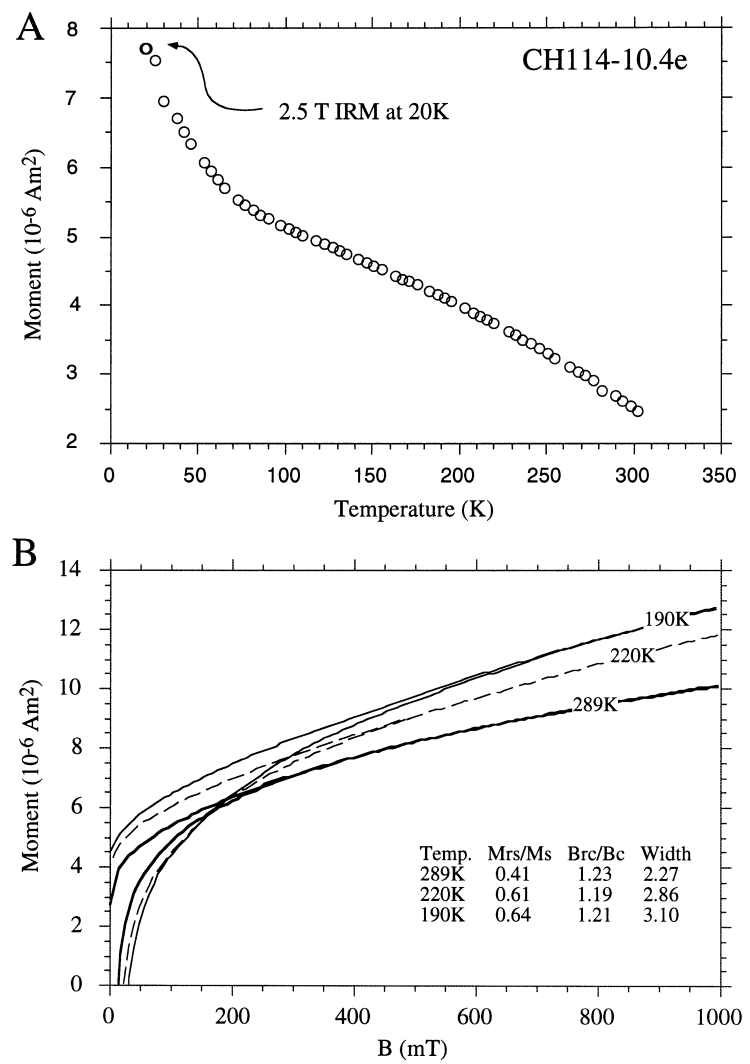

Fig. 4. Low-temperature magnetic measurements from a basalt sample from the northern EPR (see [7] for additional data on this sample). (A) Decay of a low-temperature $(20 \mathrm{~K})$ isothermal remanence (IRM; $2.5 \mathrm{~T}$ applied field) during warming to room temperature. (B) Hysteresis loops (not slope corrected) for this sample at selected temperatures.

sample contains titanomagnetites with a composition near TM55. This sample was taken from a depth of 3-4 $\mathrm{cm}$ below the chilled margin, and its hysteresis properties $\left(M_{\mathrm{rs}} / M_{\mathrm{s}}=0.41 ; B_{\mathrm{rc}} / B_{\mathrm{c}}=1.23\right)$ suggest that it contains a mixture of SD and MD grains. Together with the presence of skeletal grains up to $\sim 10 \mu \mathrm{m}$, it therefore seems unlikely that this sample would include a volumetrically significant proportion of SP grains. Yet more than two thirds of the saturation remanence acquired at $15 \mathrm{~K}$ decays during warming to room temperature.

To investigate the cause of this remanence decay, we also measured hysteresis loops for this sample at several temperatures below room temperature (Fig. 4B). The sample was cooled by nitrogen gas from the top of a liquid nitrogen dewar, delivered via a long cylindrical dewar designed to enclose the sample during a standard hysteresis measurement on the Micromag 2900. Temperatures were measured with a thermocouple placed in the gas stream directly beneath the sample. Reproducible results were obtained from room temperature to approximately $-100^{\circ} \mathrm{C}(173 \mathrm{~K})$, when the increased gas flow began causing significant disruption. Saturation magnetization values for a $\mathrm{Ni}$ standard measured over this same temperature range showed no systematic variation and provided consistent results (to within $\pm 3 \%$ ). Results from this basalt sample indicate that $B_{\mathrm{c}}, M_{\mathrm{rs}}$ and $M_{\mathrm{rs}} / M_{\mathrm{s}}$ all systematically increase with decreasing temperature (Fig. 4B). Interestingly, the degree of loop constriction, as measured by the wasp-waisted factor, increases from 2.27 at room temperature to 3.10 at $-83^{\circ} \mathrm{C}(190 \mathrm{~K})$.

Although these temperature-dependent variations in hysteresis parameters might at first glance be interpreted in terms of quenching of SP grains, temperature-dependent variations in magnetic anisotropy provide an alternative interpretation. Hodych [27] documented similar changes in hysteresis properties for two basalt samples, containing $200 \mu \mathrm{m}$ unexsolved titanomagnetite grains, over a range of temperatures from $105 \mathrm{~K}$ to room temperature. Based on the similarity of the observed coercivity and changes in magnetocrystalline and magnetoelastic anisotropy with temperature [28], Hodych concluded that changes in the anisotropy constants were responsible for the increase in $H_{\mathrm{c}}$ and $M_{\mathrm{rs}} / M_{\mathrm{s}}$ at low temperatures. We suggest that this anisotropy-related increase in remanence at low temperature provides a more plausible explanation for the decay of lowtemperature IRM in the coarse TM55 grains of the sample shown in Fig. 4 than does the unblocking of SP grains. The nearly linear decay of remanence between approximately $70 \mathrm{~K}$ and room temperature is difficult to reconcile with a dominant contribution from SP material. Indeed, the remanence loss during warming for this sample is similar to that observed for coarse-grained, Ti-rich synthetic titanomagnetites [29].

If anisotropy-related changes in remanence are important in the decay of remanence in multidomain materials, at least for Ti-rich titanomagnetites [28], we expect that systematic changes should be 
observed in the decay of a low-temperature IRM as a function of depth (and hence grain size) within a submarine lava. To test this prediction, we resampled the pillow lava shown in Fig. 2 (ph93-1) at approximately a $2 \mathrm{~mm}$ interval for the outer $20 \mathrm{~mm}$, with somewhat coarser sampling toward the pillow interior. Curie temperature data from this sample indicate a uniform Ti-rich composition for all specimens $>2 \mathrm{~cm}$ from the chilled margin $\left(T_{\mathrm{c}}=107^{\circ} \mathrm{C} \pm 7^{\circ} \mathrm{C}\right.$; $n=14)$. The remaining specimens have slightly elevated Curie temperatures $\left(125^{\circ} \mathrm{C}-170^{\circ} \mathrm{C}\right.$, with minor Curie points near $350^{\circ} \mathrm{C}$ and $550^{\circ} \mathrm{C}$ ) that we interpret as reflecting oxidation of the Ti-rich composition determined for the pillow interior. Each of these specimens was given an IRM (1.0 T field) at $77 \mathrm{~K}$ and the percentage of remanence lost after warming to room temperature was determined.

The outermost two samples near the chilled margin show approximately a $50 \%$ loss of remanence (Fig. 5A). The reduced $M_{\mathrm{rs}} / M_{\mathrm{s}}$ values together with the substantial remanent coercivities (Fig. 5B,C) of these samples suggest that unblocking of SP grains provides a reasonable explanation for this loss of remanence. The increase in the ratio of low field susceptibility to saturation magnetization (Fig. 5D) as the chilled margin is approached provides additional support for the presence of SP material in these outer samples [30]. In contrast, samples from approximately $1 \mathrm{~cm}$ below the chilled margin have hysteresis parameters indicative of stable SD grains and these samples show relatively little (15-25\%) decay of the low-temperature IRM. Samples taken at depths of $2-15 \mathrm{~cm}$ from the chilled margin have uniformly low $M_{\mathrm{rs}} / M_{\mathrm{s}}(\sim 0.32)$ suggestive of coarser (PSD-MD) grains. These samples all exhibit remanence losses during warming that are comparable to, or larger than, that observed for the finest-grained sample near the chilled margin.

These data provide strong support for the importance of anisotropy-related remanence loss in samples with PSD-MD grains. It appears that coarser grains of Ti-rich titanomagnetite may exhibit lowtemperature behavior similar to that expected from SP grains, as noted by Moskowitz et al. [29]. The use of such low-temperature remanence loss data in granulometric studies of titanomagnetite-bearing samples is therefore only credible if independent information on the likely presence of SP or MD grains

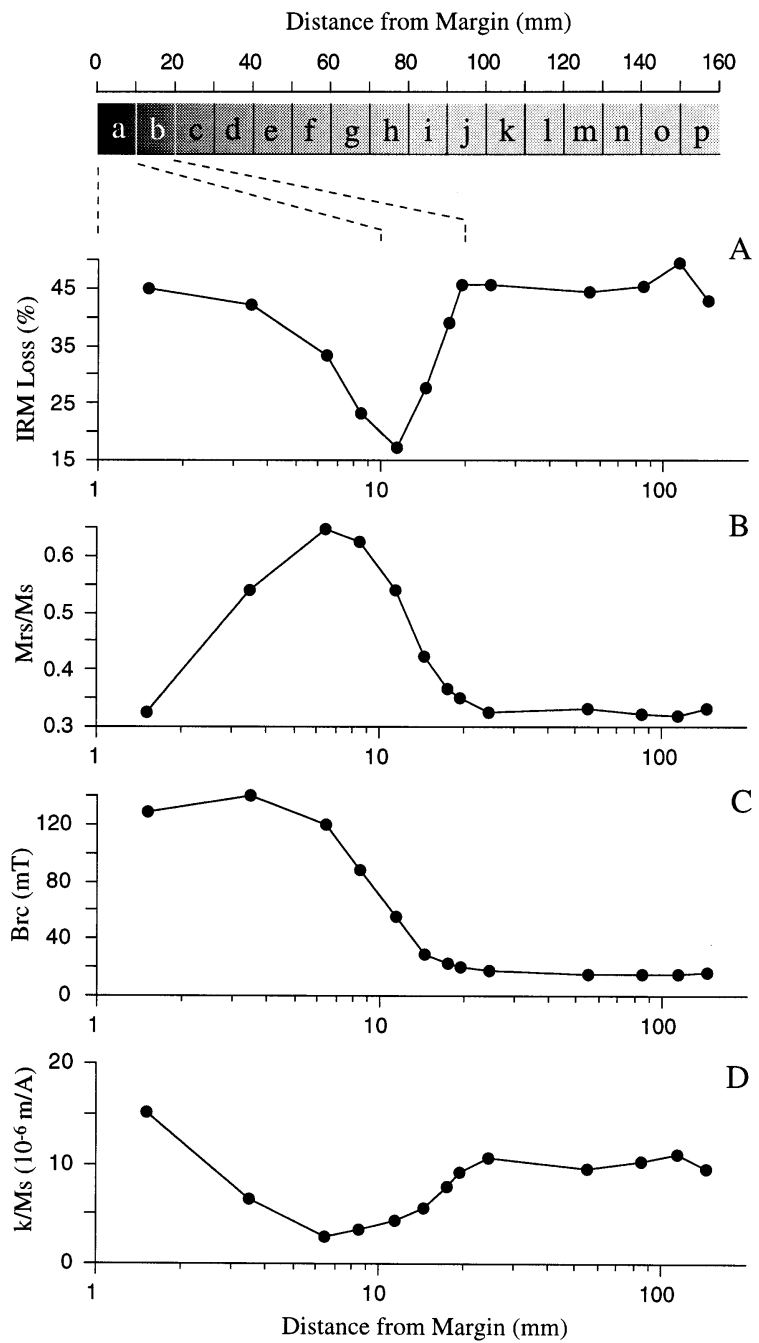

Fig. 5. Variation in magnetic properties with respect to chilled margin of sample ph93-1 (see also Fig. 2). (A) Percentage of remanence (1 T IRM at $77 \mathrm{~K}$ ) lost during subsequent warming to room temperature. Variation of (B) $M_{\mathrm{rs}} / M_{\mathrm{s}}$ and (C) $B_{\mathrm{rc}}$ illustrate location of fine-grained (SD) region near $10 \mathrm{~mm}$ and influence of SP grains nearer the margin. (D) Variation in ratio of susceptibility to $M_{\mathrm{S}}$ provides an additional indication of SP material (see [30]) near the margin. Position of $\sim 1 \mathrm{~cm}$ subsamples (labelled alphabetically from $a$ nearest the chilled margin) shown schematically in uppermost panel.

is available. In the case of submarine lavas, relative variations in grain size may be inferred from detailed sampling and trends in magnetic parameters relative to the chilled margin. The interpretation of low-temperature IRM decay in other situations must rely on 
alternative means (e.g., susceptibility/ $M_{\mathrm{s}}$ ratios) of establishing whether the grain size population contains SP or MD material. Moreover, information on the composition of the magnetic carriers (a parameter that is often difficult to determine in geological materials) is essential to obtain meaningful estimates of magnetic grain sizes from such low-temperature measurements.

\section{Temporal variations in magnetic grain size in MORB}

We are now in a position to return to the question of whether temporal changes in the average grain size of submarine lavas are discernible. The original proposition that fine-grained SP-SD material was progressively lost over time [9] relied heavily on the estimates of the contribution of ultrafine material. As illustrated above, both the shape of hysteresis loops (wasp-waisted factor) and the decay of lowtemperature IRM provide ambiguous results unless independent evidence of a volumetrically significant portion of SP (or MD) grains is available. While TEM observations provide direct evidence for the presence of ultrafine material in their youngest samples studied by $\mathrm{Xu}$ et al. [9], petrographic evidence indicates that more than $90 \%$ (by volume) of the FeTi oxides in these samples have sizes from 1 to 10 $\mu \mathrm{m}$ [9]. Simple volume considerations suggest that unimodal grain size distributions encompassing such PSD-MD grains are unlikely to have a volumetrically significant amount of SP material. For spherical grains, on the order of $10^{7} \mathrm{SP}$ particles are required to balance the saturation magnetization from a single $10 \mu \mathrm{m}$ grain. Therefore, the presence of larger grains makes it unlikely that a contribution from SP grains would be detected in bulk magnetic properties such as hysteresis or low-temperature IRM. As the presence of these coarser magnetic grains provides an alternative explanation for the rock magnetic data presented by Xu et al. [9], these data do not provide compelling evidence of changes in the contribution of SP-SD grains with time.

The rather substantial number of specimens in the present collection allows a more complete evaluation of any temporal variation in the average grain size of submarine lavas (Fig. 6). A specific predic- tion of the $\mathrm{Xu}$ et al. model is that average grain size of MORB, as measured by hysteresis parameters $\left(M_{\mathrm{rs}} / M_{\mathrm{s}}\right)$, should decrease with age as the finest magnetic grains are progressively dissolved. Zero age samples from the SEPR have a wide range of $M_{\mathrm{rs}} / M_{\mathrm{s}}$ values, from less than 0.2 to about 0.7 . A considerable portion of this range can be observed within individual pillows, emphasizing the importance of cooling history as the initial control on grain size and the need for detailed sampling to obtain representative values of magnetic properties. Samples from the Phoenix and DSDP/ODP slab collections also show large range in $M_{\mathrm{rs}} / M_{\mathrm{s}}$ values.

There is no discernible trend with age at least out to $30 \mathrm{Ma}$ (Fig. 6) and, as evidenced by the near constancy of maximum $M_{\mathrm{rs}} / M_{\mathrm{s}}$ ratios, no reason to suppose that the finest grains (i.e., those expected to have the highest $M_{\mathrm{rs}} / M_{\mathrm{s}}$ values) have been progressively dissolved over this time interval. Hysteresis data for samples from several Atlantic DSDP sites [20] provide additional support for the near constancy of maximum $M_{\mathrm{rs}} / M_{\mathrm{s}}$ over the past $\sim 30 \mathrm{Ma}$, recalling that these samples did not preferentially sample the finest-grained samples. There are very few samples with hysteresis data from older oceanic crust. On basis of our data alone, one might consider a possible decrease in $M_{\mathrm{rs}} / M_{\mathrm{s}}$ at ages $>30$ Ma (Fig. 6). However, given the appreciable scatter within individual flow units such an interpretation would be speculative at best. Moreover, magnetic hysteresis data from ODP Hole 801C that penetrated Jurassic crust include values of $M_{\mathrm{rs}} / M_{\mathrm{s}}$ above 0.6 [21] and provides evidence against any simple age trend.

The Xu et al. model of progressive dissolution of SD-SP grains in MORB also predicts that coercivity should decrease as a function of age. As with the $M_{\mathrm{rs}} / M_{\mathrm{s}}$ data, the remanent coercivities for the zero age SEPR samples span a large range (Fig. 6B) with some $B_{\mathrm{rc}}$ values extending to over $100 \mathrm{mT}$. The Phoenix samples extend to somewhat higher values of $B_{\mathrm{rc}}$ of about $200 \mathrm{mT}$ whereas the older $(>1 \mathrm{Ma}$ ) DSDP/ODP samples tend to have somewhat reduced maximum values of $B_{\mathrm{rc}}$ of $100 \mathrm{mT}$ or even less. Unfortunately, Wallick and Steiner [21] did not report $B_{\mathrm{rc}}$ values from Jurassic Hole 801C. A systematic decrease in $B_{\mathrm{rc}} / B_{\mathrm{c}}$ at ages greater than 30 Ma cannot be precluded; however, the demonstration of a con- 

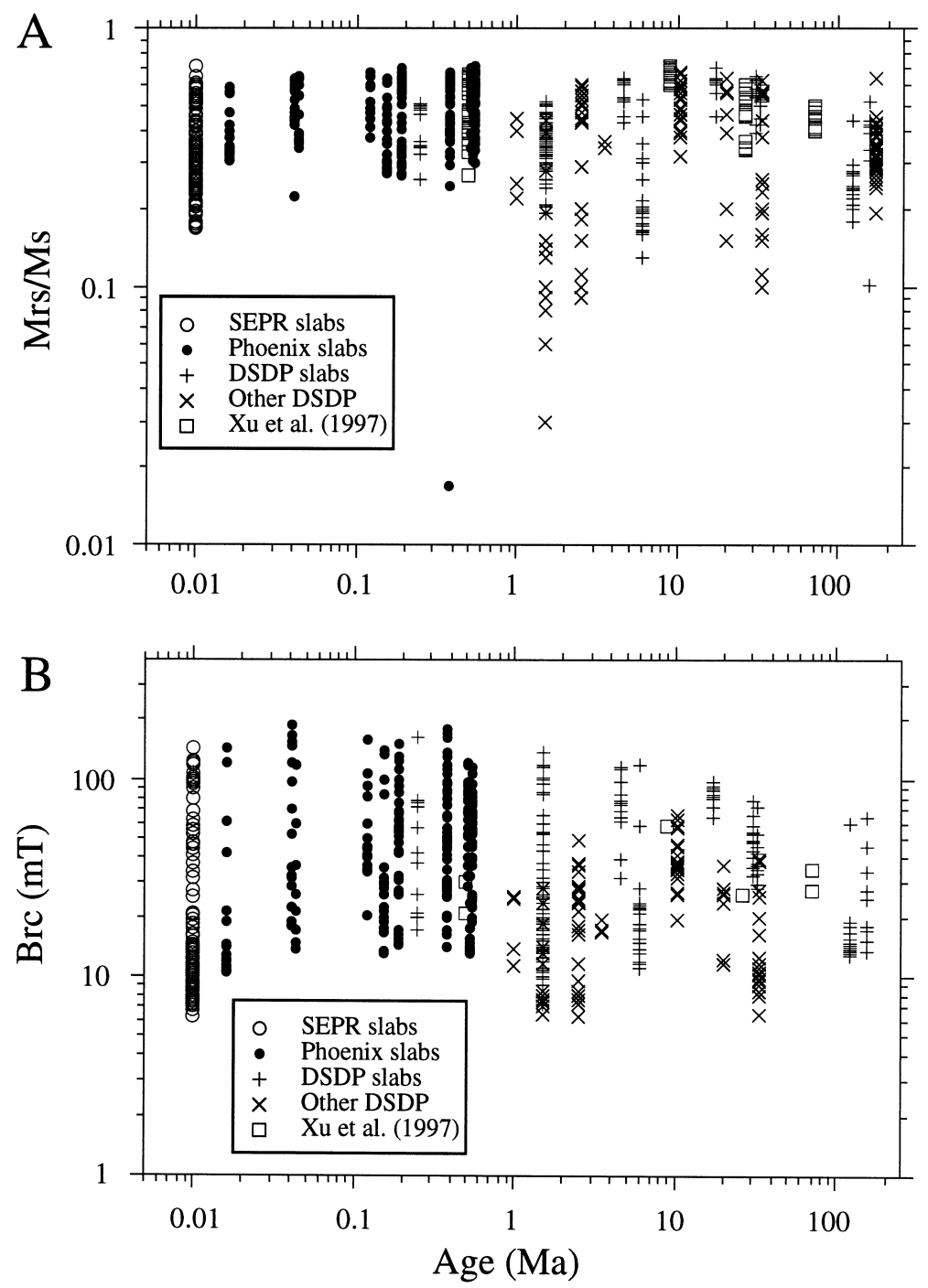

Fig. 6. Variation in (A) $M_{\mathrm{rs}} / M_{\mathrm{s}}$ and (B) $B_{\mathrm{rc}}$ with age in submarine basalt samples. Symbols as in Fig. 1 .

vincing trend will likely require a substantial amount of additional data given the scatter in individual flow units.

\section{Discussion and conclusions}

The rapid cooling of submarine lavas results in substantial variations in the average magnetic grain size over small spatial scales $(\sim 1 \mathrm{~cm})$ near the chilled margin. This variability constitutes a sig- nificant obstacle to establishing temporal trends or assessing processes affecting the magnetization of submarine lavas unless spatial variations within individual flows are carefully documented. Fortunately, trends in the average grain size variation in MORB samples are generally well predicted by distance from the cooling margin. Use of this independent grain size information allows the recognition of MD-SD and SD-SP mixing trends on a Day-plot of hysteresis parameters. Samples from the interiors of submarine flows/pillows have hysteresis ratios com- 
patible with a mixing trend between MD-like grains and an SD endmember. Only the finest-grained samples (typically within $1-2 \mathrm{~cm}$ of the glassy margin) have volumetrically significant proportions of SP grains, delineating a second shallower SD-SP trend on the bilogarithmic Day-plot. This trend is compatible with mixing between a SD endmember with dominant cubic anisotropy $\left(M_{\mathrm{r}} / M_{\mathrm{S}}=0.83-0.87\right.$; $B_{\mathrm{rc}} / B_{\mathrm{c}}=1.04-1.08$; [15]) and an empirically determined SP endmember with characteristically high $B_{\mathrm{rc}} / B_{\mathrm{c}}(\sim 100)$ and low $M_{\mathrm{rs}} / M_{\mathrm{s}}(\sim 0.02)$.

We suggest that the most promising routine criteria for magnetic granulometry are the MD-SD and SD-SP mixing trends in hysteresis ratios on a Dayplot. The mixing trend between SD and SP grains established here relies on the systematic variation in magnetic grain size imposed by cooling in submarine lavas. Although the slope of this trend may be appropriate only for titanomagnetite compositions, a similar (though slightly shallower) trend has also been established for mixtures of SD and SP magnetite in carbonates [30,31]. In these samples, elevated susceptibility to saturation magnetization ratios provide independent evidence for a large component of ultrafine SP material. In addition, well characterized submarine basaltic glass samples (with a maximum observed grain size near the SD-SP boundary) have hysteresis ratios that are entirely compatible with the SD-SP mixing trend suggested here [3,22]. Recognition of these mixing trends should allow improved estimates of magnetic grain size in submarine lavas, but also may prove applicable in other settings as well.

The validity of granulometric interpretations, at least in titanomagnetite-bearing samples, apparently requires some independent information on whether coarse (MD) or ultrafine material is likely to be present. For example, two common rock magnetic techniques for assessing the contribution of SP material (low-temperature IRM decay and hysteresis loop constriction) may provide ambiguous results. Detailed sampling of a submarine flow demonstrates that decay of a low-temperature IRM may be characteristic of SP grains, but this same behavior also occurs in samples where such ultrafine grains are unlikely to be present in volumetrically significant amounts (Fig. 5). The temperature-dependent variation of magnetocrystalline and magnetoelastic an- isotropy in multidomain titanomagnetite grains may produce an equivalent, or larger, remanence loss than that associated with SP material.

Similarly, constriction of hysteresis loops alone is a poor indicator of the presence of SP grains. Because significant loop constriction occurs in samples from pillow interiors, and whose hysteresis parameters lie towards the MD endmember, we suggest that bimodal mixtures of coarse (MD) and SD grains may in some cases provide the coercivity contrast necessary to produce wasp-waisted loops. In addition, we note that loop constriction (at least as measured by the wasp-waisted factor in [9]) is an intrinsic characteristic of materials with dominant magnetocrystalline anisotropy. Model hysteresis loops for SD magnetocrystalline materials [32] yield wasp-waisted factors of 1.08 and 1.13 for $K_{1}<0$ and $K_{1}>0$, respectively. Moreover, oxidation of titanomagnetites can apparently produce more significant degrees of hysteresis loop constriction. Highly oxidized synthetic monodomain titanomagnetites $\left(\mathrm{Fe}_{2.4} \mathrm{Ti}_{0.4} \mathrm{Al}_{0.2} \mathrm{O}_{4}\right)$ had hysteresis loops with a wasp-waisted factor of approximately 1.4 [26]. As these highly oxidized $(z>0.9)$ grains showed evidence of a small proportion of hematite, Özdemir and Dunlop [26] attributed the constricted loops to exchange or magnetostatic coupling between these magnetically hard and soft phases. Although the mechanism for the pronounced constriction in hysteresis loops from submarine lavas is uncertain, dominant magnetocrystalline anisotropy, oxidation, and possibly mixtures of $\mathrm{MD}$ and $\mathrm{SD}$ grains all provide plausible mechanisms for generating the required coercivity contrast.

Examination of hysteresis data from a large number $(\sim 750)$ of samples spanning a range of ages from $\sim 0$ to 122 Ma provides no evidence for systematic temporal changes in the average magnetic grain size in submarine lavas. Although there is substantial variability of grain size within individual flows, no discernible change in $M_{\mathrm{rs}} / M_{\mathrm{s}}$ is found with age, at least to $30 \mathrm{Ma}$. Moreover, maximum $M_{\mathrm{rs}} / M_{\mathrm{s}}$ values $>0.5$ in samples from Jurassic age crust [21] suggest that fine-grained material is preserved over much longer time scales, although hysteresis data from older sea floor basalts are scarce. We conclude that, even with the much larger data set available here, there is no convincing evidence of preferential 
dissolution of the finest magnetic grains with time as hypothesized by $\mathrm{Xu}$ et al. [9]. This does not mean that magnetic properties do not change with time, but rather that the average magnetic grain size seems to preserve the overall distribution from initial cooling. For example, the apparent lack of an age-dependent trend in $M_{\mathrm{rs}} / M_{\mathrm{s}}$ does not preclude alteration, since hysteresis ratios are not sensitive to compositional variations (provided the dominant anisotropy remains the same). Given the large variability within individual flows, detection of temporal variations in the average grain size of submarine lavas is likely to require a substantially larger number of well documented samples.

\section{Acknowledgements}

We thank R. Batiza, J. Sinton, J. Mahoney, C. Langmuir, and J. Bender for providing access to dredged samples and the Ocean Drilling Program for providing access to the DSDP cores. We thank L. Tauxe for valuable discussions and S. Banerjee and R. Merrill for providing thoughtful reviews. We thank S. Brachfeld, S. Banerjee, M. Jackson for providing the low-temperature remanence data shown in Fig. 4A. Support for this work was provided through NSF grants (OCE94-16839 (DVK), OCE95-03916 (JG) and EAR95-27533 (L. Tauxe, JG). LamontDoherty Earth Observatory contribution 5906. [MK]

\section{References}

[1] G.M. Smith, The magnetic structure of the marine basement, Aquat. Sci. 2 (1990) 205-227.

[2] T. Pick, L. Tauxe, Holocene paleointensities: Thellier experiments on submarine basaltic glass from the East Pacific Rise, J. Geophys. Res. 98 (1993) 17949-17964.

[3] T. Pick, L. Tauxe, Characteristics of magnetite in submarine basaltic glass, Geophys. J. Int. 119 (1994) 116-128.

[4] D.V. Kent, J. Gee, Magnetic alteration of zero-age oceanic basalt, Geology 24 (1996) 703-706.

[5] M. Marshall, A. Cox, Magnetism of pillow basalts and their petrology, Geol. Soc. Am. Bull. 82 (1971) 537-552.

[6] P.J.C. Ryall, J.M. Ade-Hall, Radial variation of magnetic properties in submarine pillow basalt, Can. J. Earth Sci. 12 (1975) 1959-1969.

[7] D.V. Kent, J. Gee, Grain size dependent alteration and the magnetization of oceanic basalts, Science 265 (1994) 1561-1563.
[8] M.M. Bina, M. Prévot, Thermomagnetic investigations of titanomagnetite in submarine basalts: evidence for differential maghemitization, Phys. Earth Planet. Inter. 54 (1989) 169-179.

[9] W. Xu, R. Van der Voo, D.R. Peacor, R.T. Beaubouef, Alteration and dissolution of fine-grained magnetite and its effects on magnetization of the ocean floor, Earth Planet. Sci. Lett. 151 (1997) 279-288.

[10] U. Bleil, N. Petersen, Variations in magnetization intensity and low-temperature titanomagnetite oxidation of ocean floor basalts, Nature 301 (1983) 384-388.

[11] W.X. Xu, D.R. Peacor, W.A. Dollase, R. Van der Voo, R. Beaubouef, Transformation of titanomagnetite to titanomaghemite: a slow, two-step, oxidation-ordering process in MORB, Am. Mineral. 82 (1997) 1101-1110.

[12] J. Gee, D.V. Kent, Magnetization of axial lavas from the southern East Pacific Rise $\left(14^{\circ}-23^{\circ} \mathrm{S}\right)$ : geochemical controls on magnetic properties, J. Geophys. Res. 102 (1997) 24873-24886.

[13] R. Batiza, Y. Niu, J.L. Karsten, W. Boger, E. Potts, L. Norby, R. Butler, Steady and non-steady state magma chambers below the East Pacific Rise, Geophys. Res. Lett. 23 (1996) 221-224.

[14] R. Day, M.D. Fuller, V.A. Schmidt, Hysteresis properties of titanomagnetites: grain-size and compositional dependence, Phys. Earth Planet. Inter. 13 (1977) 260-267.

[15] E.C. Stoner F.R.S., E.P. Wohlfarth, A mechanism of magnetic hysteresis in heterogeneous alloys, Philos. Trans. R. Soc. London, Ser. A 240 (1948) 599-642.

[16] J. Gee, D.V. Kent, Magnetic hysteresis in young mid-ocean ridge basalts: dominant cubic anisotropy?, Geophys. Res. Lett. 22 (1995) 551-554.

[17] D.J. Dunlop, Ö. Özdemir, Rock Magnetism Fundamentals and Frontiers, Cambridge Univ. Press, Cambridge, 1997, $573 \mathrm{pp}$.

[18] D.J. Dunlop, Hysteresis properties of magnetite and their dependence on particle size: a test of pseudo-single-domain remanence models, J. Geophys. Res. 91 (1986) 9569-9584.

[19] J.C. Stormer Jr., The effects of recalculation on estimates of temperature and oxygen fugacity from analyses of multicomponent iron-titanium oxides, Am. Mineral. 68 (1983) 586-594.

[20] R. Day, S. Halgedahl, M. Steiner, K. Kobayashi, T. Furuta, T. Ishii, A. Faller, Magnetic properties of basalts from DSDP Leg 49, Init. Rep. DSDP 49 (1978) 781-791.

[21] B.P. Wallick, M.B. Steiner, Paleomagnetic and rock magnetic properties of Jurassic quiet zone basalts, Hole 801C, Proc. ODP, Sci. Results 129 (1992) 455-470.

[22] L. Tauxe, T.A.T. Mullender, T. Pick, Potbellies, waspwaists, and superparamagnetism in magnetic hysteresis, J. Geophys. Res. 101 (1996) 571-583.

[23] T. Nagata, B.J. Carleton, Magnetic remanence coercivity of rocks, J. Geomag. Geoelectr. 39 (1987) 447-461.

[24] P.J. Wasilewski, Magnetic hysteresis in natural materials, Earth Planet. Sci. Lett. 20 (1973) 67-72.

[25] G. Muttoni, 'Wasp-waisted' hysteresis loops from a 
pyrrhotite and magnetite-bearing remagnetized Triassic limestone, Geophys. Res. Lett. 22 (1995) 3167-3170.

[26] Ö. Özdemir, D.J. Dunlop, An experimental study of chemical remanent magnetizations of synthetic monodomain titanomaghemites with initial thermoremanent magnetizations, J. Geophys. Res. 90 (1985) 11513-11523.

[27] J.P. Hodych, Magnetic hysteresis as a function of low temperature for deep-sea basalts containing large titanomagnetite grains - inference of domain state and controls on coercivity, Can. J. Earth Sci. 19 (1982) 144-152.

[28] Y. Syono, Magnetocrystalline anisotropy and magnetoconstriction of $\mathrm{Fe}_{3} \mathrm{O}_{4}-\mathrm{Fe}_{2} \mathrm{TiO}_{4}$ series, Jpn. J. Geophys. 4 (1965) 71-143.

[29] B.M. Moskowitz, M. Jackson, C. Kissel, Low-temperature magnetic behavior of titanomagnetites, Earth Planet. Sci. Lett. 157 (1998) 141-149.

[30] M. Jackson, P. Rochette, G. Fillion, S. Banerjee, J. Marvin, Rock magnetism of remagnetized Paleozoic carbonates: low-temperature behavior and susceptibility characteristics, J. Geophys. Res. 98 (1993) 6217-6225.

[31] M. Jackson, Diagenetic sources of stable remanence in remagnetized Paleozoic cratonic carbonates: a rock magnetic study, J. Geophys. Res. 95 (1990) 2753-2762.

[32] M. Walker, P.I. Mayo, K. O’Grady, S.W. Charles, R.W. Chantrell, The magnetic properties of single-domain particles with cubic anisotropy, I, hysteresis loops, J. Phys. Condens. Matter 5 (1993) 2779-2792. 